\title{
Fall, M. Maltais, D. et Tremblay, S. (dir) (2017). Vivre-ensemble dans les régions du Québec : défis et enjeux contemporains Chicoutimi, Québec : Groupe de recherche et d'intervention régionales, $82 \mathrm{p}$.
}

\section{Christian Bélanger ${ }^{\mathrm{a}}$}

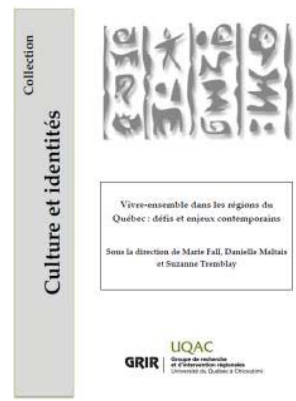

Cet ouvrage collectif contribue à l'actualisation des travaux et des réflexions à propos du vivre-ensemble. Phénomène social d'importance, il est l'un des nombreux défis qu'accentue la mondialisation. Le vivre-ensemble soulève un certain nombre d'enjeux propres aux communautés ainsi qu'aux organisations. La plupart du temps, ce dernier reste toutefois un objet étudié dans le contexte des grands pôles urbains. Le présent ouvrage se démarque donc des travaux qui ont pu le précéder : il traite, en effet, du vivre-ensemble en fonction de la situation ayant prévalence au SaguenayLac-Saint-Jean.

Produit à la suite de la tenue du colloque du Groupe de recherche et d'intervention régionales (GRIR) de l'Université du Québec à Chicoutimi (UQAC) en 2016, Vivre-

ensemble dans les régions du Québec : défis et enjeux contemporains, l'ouvrage présente les contributions de huit intervenants. L'introduction de Marie Fall met en lumière l'importance de ce colloque dans le cadre des préoccupations sociales contemporaines. Parmi celles-ci, l'accentuation des préoccupations sécuritaires a contribué à la création d'un climat de tension permanent et à la polarisation de la population. Cela a eu pour incidence de favoriser la tentation du repli identitaire ainsi que le rejet de la différence. À ces préoccupations s'ajoutent également le renforcement et la présence de discours populistes au sein de l'espace public, de même que la réalité spécifiquement québécoise des débats découlant de la présentation d'un projet de loi sur la laïcité (mieux connu sous l'appellation de Charte des valeurs).

L'ouvrage collectif comprend un total de six textes. Le premier d'entre eux est de Dominique Payette. Il s'intéresse à l'incidence des radios d'opinion de la région de Québec. Celles-ci contribuent à influencer ainsi qu'à orienter certains des débats prenant place dans l'espace public. N'étant plus des médias généralistes, s'adressant à des auditoires spécialisés, ces radios renforcent la particularisation de ces demiers par l'offre d'une opinion en ce sens. Ce qui n'est pas sans effet sur la qualité du débat politique et de la perpétuation de certains préjugés ou représentations sociales attenantes à certains groupes de la population.

Deux des textes de l'ouvrage s'attardent à des réalités vécues par les populations autochtones. Le premier d'entre eux est de Camil Girard. À l'aide de l'analyse du philosophe Michel Foucault, ce dernier met en évidence l'exclusion dont ces populations ont été victimes dans le cadre de l'acte de fondation du Canada en 1867. Loin d'être le fruit du hasard, celle-ci fut plutôt l'objet d'un long processus dont la nature peut être exposée. Le deuxième texte est de Julie Rousseau. Conseillère déléguée à Mashteuiatsh lors de la tenue du colloque, elle présente sa vision de ce que devrait être le vivre-ensemble entre autochtones et allochtones. Ce dernier implique l'existence d'un respect mutuel entre les deux groupes. Elle en profite également pour mettre en évidence les réalités propres au vécu des Pekuakamiulnuatsh, de même que les défis liés à leur développement, et à la préservation de leur culture et de leur mode de vie.

a Chargé de cours, Ph. D., Université du Québec à Chicoutimi 
La situation des travailleuses et travailleurs étrangers temporaires (TET) au Saguenay-Lac-Saint-Jean fait l'objet d'un texte de Jorge Frozzini. Cet auteur présente certaines des particularités structurelles du nouveau programme de travailleurs étrangers temporaires. Celles-ci concernent notamment sa division en deux programmes distincts, la présence de nouvelles catégories de travailleurs, de même que les éléments de différenciation entre ces dernières. Cela permet de faire ressortir les facteurs de précarisation des conditions de vie de ces travailleuses, travailleurs, et de leurs familles, de même que les obstacles qui limitent leur participation à la société d'accueil.

Les réalités propres à la fondation et à l'existence d'un collectif interculturel et interreligieux de la part de citoyennes et de citoyens voulant favoriser le vivre-ensemble sont l'objet du texte de Jocelyn Girard et Christian Bélanger. Faisant un bilan de l'action menée par le collectif Coexister au Saguenay-Lac-Saint-Jean, fondé en 2013 à la suite d'un acte de vandalisme commis à l'encontre de la mosquée de Saguenay, ceux-ci présentent les différents événements ayant ponctué l'existence de cette organisation. Ils s'attardent également à l'analyse des conditions d'existence, de même qu'aux perspectives de pérennité de ce type d'organisation citoyenne.

Pour sa part, le texte de Siegfried L. Mathelet s'attarde à la relation des notions de vivre-ensemble et de lutte antiraciste en contexte québécois. Il met en évidence la crise que traverse l'interculturalisme en tant qu'idéologie orientant l'action de l'État dans l'élaboration de politiques permettant de lutter contre le racisme. Son analyse l'amène à constater la nécessité d'adapter les actions allant en ce sens en fonction du contexte des processus et des phénomènes de racialisation. Celle-ci appelle également au dépassement de l'approche essentiellement culturelle et religieuse du vivre-ensemble et de la lutte antiraciste.

Vivre-ensemble dans les régions du Québec : défis et enjeux contemporains est un ouvrage de vulgarisation pertinent pour tout acteur intéressé à développer ses connaissances à propos du vivre-ensemble. Autant les gestionnaires appelés à œuvrer à la mise en place d'approches, de mesures, voire de politiques allant dans le sens du vivre-ensemble, que les chercheurs voulant amorcer des réflexions plus approfondies à son propos peuvent y trouver leur compte. 